\title{
Implementation and evaluation of a COVID-19 rapid follow-up service for patients discharged from the emergency department
}

\author{
Authors: Lucy CK Bell, ${ }^{\mathrm{A}}$ Caitlin Norris-Grey, ${ }^{\mathrm{B}}{ }^{\mathrm{A}}$ Akish Luintel, ${ }^{\mathrm{A*}}$ Gabriella Bidwell, ${ }^{\mathrm{A}}$ David Lanham, ${ }^{\mathrm{C}}$ \\ Michael Marks, ${ }^{D}$ Tim Baruah, ${ }^{\mathrm{E}}$ Luke O'Shea, ${ }^{\mathrm{F}}$ Melissa Heightman ${ }^{\mathrm{G}}$ and Sarah Logan; ${ }^{\mathrm{H}}$ on behalf of the University \\ College London Hospitals COVID response team
}

The COVID-19 pandemic has necessitated rapid adaptation of healthcare providers to new clinical and logistical challenges. Following identification of high levels of emergency department (ED) reattendance among patients with suspected COVID-19 at our centre, we piloted a rapid remote follow-up service for this patient group. We present our service framework and evaluation of our pilot cohort of 192 patients. We followed up patients by telephone within 36 hours of their ED attendance. Pulse oximetry was used for remote monitoring of a subset of patients. Patients required between one and six consecutive telephone assessments, dependent on illness severity, and 23 patients were recalled for in-person assessment. Approximately half of patients with confirmed or probable COVID-19 required onward referral for respiratory follow-up. This framework reduced unplanned ED reattendances in comparison with a retrospective comparator cohort ( $4.7 \%$ from $22.6 \%$ ). We reproduced these findings in a validation cohort with a high prevalence of acute COVID-19, managed through the clinic in September-October 2020, where we identified an unplanned ED reattendance rate of $5.2 \%$. We propose that rapid remote follow-up is a mechanism by which ambulatory patients can be clinically supported during the acute phase of illness, with benefits both to patient care and to health service resilience.

KEYWORDS: COVID-19, infectious diseases, ambulatory care, emergency medicine, service evaluation

DOI: $10.7861 /$ clinmed.2020-0816

Authors: Ainfectious diseases registrar, Hospital for Tropical Diseases, London, UK; ${ }^{B}$ medical student, Hospital for Tropical Diseases, London, UK; ' Cacute medicine registrar, University College Hospital, London, UK; ' Dinfectious diseases consultant, Hospital for Tropical Diseases, London, UK and London School of Hygiene \& Tropical Medicine, London, UK; Eemergency medicine consultant, University College Hospital, London, UK; F Firector of innovation, University College Hospital, London, UK; Grespiratory medicine consultant, University

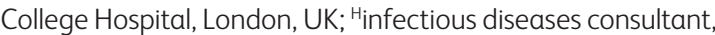
Hospital for Tropical Diseases, London, UK; * joint first authors

\section{Clinical problem}

Health systems globally have faced enormous clinical and logistical challenges during the COVID-19 pandemic.' The decision to admit or discharge a patient with suspected COVID-19 from the emergency department (ED) is a key point in an individual patient journey, with implications for outcome, infection control and hospital capacity. In the context of an emerging infectious disease such as COVID-19, uncertainty about trajectory and risk of respiratory deterioration in the second week of illness ${ }^{2}$ contributes to challenges surrounding safe discharge.

Outcomes of a cohort of patients discharged from the ED with suspected COVID-19 during April 2020 were evaluated by colleagues at our centre. ${ }^{3}$ They identified a high ED reattendance rate ( 45 of 199 patients; $22.6 \%$ ), with a significant minority of patients requiring admission ( 12 of $199 ; 6.0 \%$ ). Structured telephone follow-up identified many patients with persistent breathlessness following acute illness, in keeping with reports of 'long-tail' COVID-19 symptoms ${ }^{4,5}$ and with evidence from other respiratory viruses. ${ }^{6-8}$ These results prompted us to identify a clinical need for a remote follow-up service for patients discharged from the ED with suspected COVID-19. Our aims were that this service would support patient self-management in the community, proactively identify deteriorating patients requiring reassessment, and form a pathway via which patients with persistent symptoms could be referred into specialist follow-up. We also devised the service as a mechanism by which patients who were tested for SARS-CoV-2 during ED attendance could be provided with their test result, contextualised with clinical and infection control advice. We present the framework of our service, evaluation of the cohort of patients managed during its pilot period in May-June 2020, and evaluation of a second validation cohort managed in a time of rising COVID-19 community prevalence in SeptemberOctober 2020. This analysis was conducted as a retrospective service evaluation project and therefore individual consent and formal ethical review were not required, as per assessment using the NHS Health Research Authority system (HRA) decision tool. ${ }^{9}$ The project was registered as a service evaluation with the Hospital for Tropical Diseases Research and Audit Committee, and the pathway was approved by the University College London Hospitals (UCLH) COVID-19 clinical guidelines committee. 


\section{ED attender with suspected COVID-19}

$>$ Safe for discharge from ED (all of): $\mathrm{O}_{2}$ saturation $\geq 94 \%$ and exercise desaturation $<2 \%, \mathrm{HR}<110, \mathrm{RR}<23$

$>$ Criteria for considering pulse oximeter (any of): CRP $>50$; RR $>20 ; \mathrm{O}_{2}$ saturation 94 or $95 \%$; exercise desaturation $>2 \%$; typical significant COVID-19 radiological abnormalities

\section{EHRS report}

$>$ Identifies all ED discharges with COVID-19-related diagnosis and/or tested for SARS-CoV-2 in the ED

$>$ Clinic doctor triages list and schedules patients for follow-up call within 36 hours of attendance

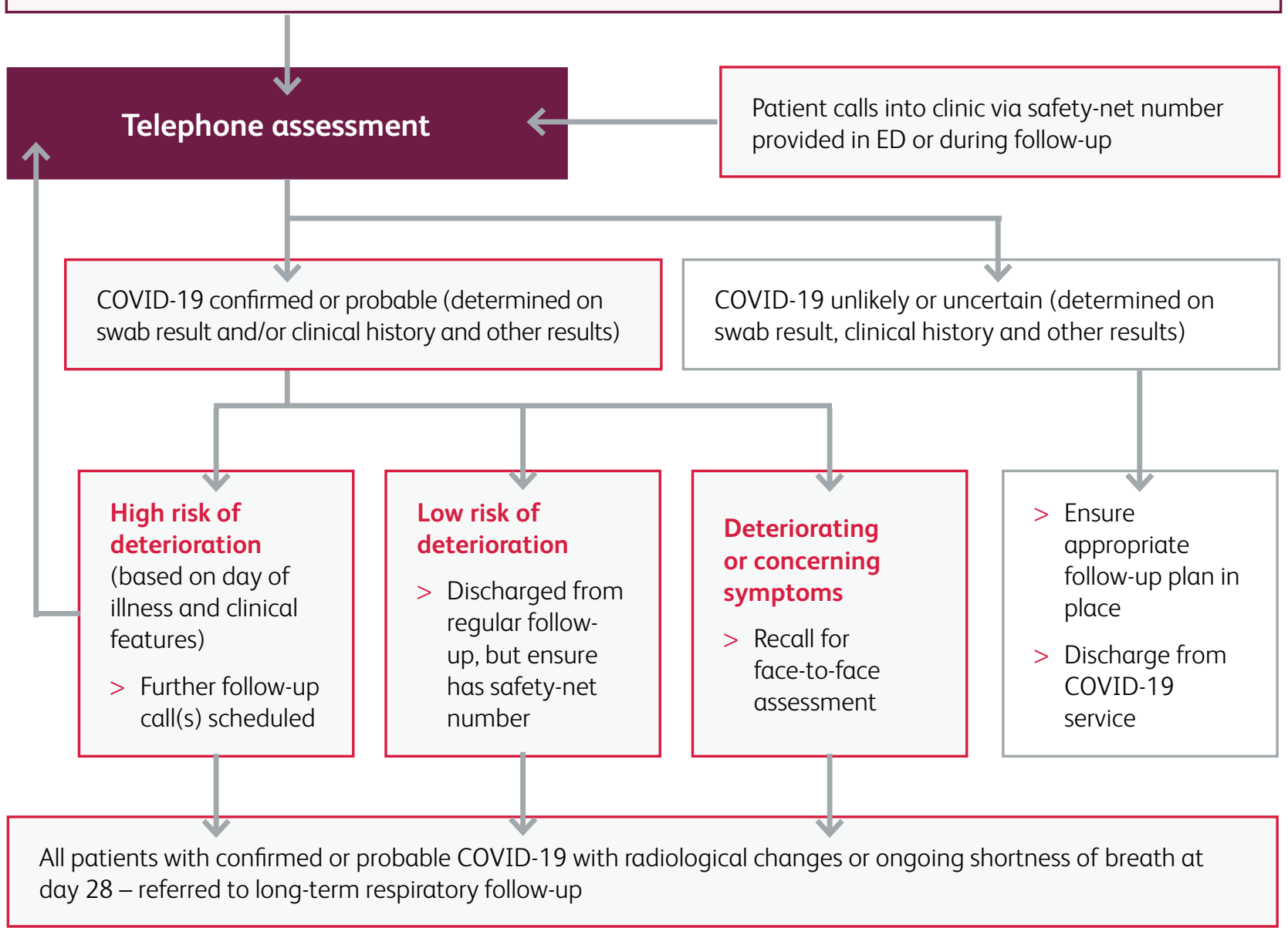

Fig 1. Rapid remote follow-up pathway. $C R P=C$-reactive protein; $E D=$ emergency department; $H R=$ heart rate; $R R=$ respiration rate.

\section{Proposed solution}

Our rapid remote follow-up pathway is shown in Fig 1. Patients with suspected COVID-19 were identified as eligible for discharge by ED clinicians based on specified criteria. A subset of these patients were eligible for provision of a pulse oximeter, on the basis of higher-risk clinical features. All patients were given an information leaflet with advice on infection control, self-monitoring, indications to reattend for assessment, and a contact phone number for clinical advice in hours.

The follow-up clinic was led by an infectious diseases specialist registrar and ran 7 days a week. An electronic health records system (EHRS) report was used daily to identify patients who had been discharged from the ED in the previous 36 hours with a COVID-19-related clinical code, and/or who had been tested for SARS-CoV-2 during attendance. This list was triaged and patients scheduled for a telephone follow-up appointment within 36 hours of attendance. A standardised electronic proforma was used for clinical documentation of assessments.

On the basis of their telephone assessment and test results, the clinic registrar allocated patients into diagnosis categories of confirmed/probable COVID-19, unlikely COVID-19 or uncertain 
diagnosis. Those with confirmed/probable COVID-19 were categorised as being at low or high risk of deterioration. Patients were scheduled for further follow-up telephone calls as judged necessary by the clinician on the basis of this assessment. Low-risk patients were not proactively followed up, but were encouraged to use the clinic's 'safety-net' phone number if needed.

Both scheduled and 'patient-activated' calls made to the clinical contact number could trigger a recall for face-to-face review if clinically indicated. Patients were assessed in a 'hot' or 'cold' clinical area of the hospital, dependent on their duration of illness and potential infectivity if $<14$ days from onset. Patients with symptoms persisting beyond 28 days, or who had COVID-19associated radiological changes at their initial attendance, were referred to a specialist COVID-19 respiratory clinic at our centre for onward management. Patients with alternative diagnoses, or those who required non-COVID-19-related investigation, were referred for alternative follow-up as indicated.

\section{Results to date}

We evaluated a cohort of 192 patients with suspected COVID-19 who were managed through our remote follow-up service during its pilot period (27 April - 3 June 2020). This comprised clinical activity of a median of 9 (range 2-17) telephone clinic appointments per day (supplementary material S1; Fig S1). Background demographics and clinical characteristics at ED presentation are shown in Table 1. Clinical features were consistent with mild illness, with vital signs within the normal range and low serum C-reactive protein levels (Table 1). Patients tended to present late in disease, with a median day of illness at presentation of 13 days (interquartile range (IQR) 3-28 days). The majority of patients fell into a low-risk (53.6\%) or medium-risk (35.9\%) category for developing moderate/severe COVID-19, on the basis of age and comorbidities (supplementary material S1; Table S1).

The majority of patients (79.7\%) were tested for SARS-CoV-2 in the ED (Table 1). Reasons for not testing patients included an existing positive result from another service or presentation $>28$ days following symptom onset. Test results and diagnoses are shown in supplementary material S1, Table S2. Twelve new COVID-19 diagnoses were made, alongside nine patients who had previously tested positive. A diagnosis of probable COVID-19 was made in a further 62 patients on the basis of their clinical characteristics, despite not being tested or having a negative test result from ED presentation (recognising the inherent limitations in test sensitivity

Table 1. Pilot cohort: patient demographics

and characteristics at emergency department presentation, 27 April - 3 June 2020

Demographics

Age, years, median (IQR)

Female, n (\%)

Never smoker, n (\%)

HCW or recent healthcare exposure, n (\%)

Lives alone, n (\%)

\section{Characteristics}

Day of illness, median (IQR)

Oxygen saturation, \%, median (IQR)

Respiratory rate, breaths per minute, median (IQR)

C-reactive protein, $\mathrm{mg} / \mathrm{L}$, median (IQR)

Chest $\mathrm{X}$-ray abnormality (any), n (\%)

D-dimer, $\mu \mathrm{g} / \mathrm{L}$, median $(\mathrm{IQR})^{\mathrm{a}}$

CTPA positive for pulmonary embolism ${ }^{\mathrm{b}}$

Swabbed for SARS-CoV-2 in ED, $\mathrm{n}$ (\%)

Missing data: smoking status (10), household status (10), C-reactive protein (16), oxygen saturation (6), respiratory rate (5) and day of illness (5). Twenty patients did not have chest $\mathrm{X}$-rays at $\mathrm{ED}$ attendance. ${ }^{\circ} \mathrm{D}$-dimer was checked in 80 patients (low risk 52; medium risk 22; high risk 6); ' 22 patients had CTPAs (low risk 10; medium risk 9; high risk 3 ). CTPA = computed tomography pulmonary angiography; $\mathrm{HCW}=$ healthcare worker; $\mathrm{IQR}=$ interquartile range.

and the effect of time after illness onset). ${ }^{10}$ As described above, diagnoses of probable COVID-19 were made by the infectious diseases clinician during telephone follow-up assessments. Either confirmed or probable COVID-19 was diagnosed in a cumulative $43.2 \%$ of the overall cohort.

Follow-up trajectories are shown in Table 2, stratified into confirmed/probable COVID-19 versus unlikely COVID-19 / uncertain diagnosis. A small number of patients $(4.2 \%)$ were provided with pulse oximeters on discharge, and other patients (10.9\%) reported using self-sourced pulse oximeters for home monitoring. The median number of telephone assessments per patient was 1

Table 2. Cohort trajectories

\begin{tabular}{|c|c|c|c|}
\hline & $\begin{array}{l}\text { All patients, } \\
n=192\end{array}$ & $\begin{array}{l}\text { Confirmed or probable } \\
\text { COVID-19, } n=83\end{array}$ & $\begin{array}{l}\text { Unlikely or uncertain } \\
\text { COVID-19, } n=109\end{array}$ \\
\hline Discharged with pulse oximeter, n (\%) & $8(4.2)$ & $6(7.2)$ & $2(1.8)$ \\
\hline Using own pulse oximeter, $\mathrm{n}(\%)$ & $21(10.9)$ & $17(20.5)$ & $4(3.7)$ \\
\hline Number of telephone encounters, median (range) & $1(1-6)$ & $1(1-6)$ & $1(1-4)$ \\
\hline Planned reattendances, n ( \% ) & $23(12.0)$ & $18(21.7)$ & $5(4.6)$ \\
\hline Planned reattendances leading to admissions, n ( \%) & $5(2.6)$ & $4(4.8)$ & $1(0.9)$ \\
\hline Unplanned reattendances, $\mathrm{n}(\%)$ & $9(4.7)$ & $6(7.2)$ & $3(2.8)$ \\
\hline Unplanned reattendances leading to admissions, $\mathrm{n}(\%$ ) & $2(1.0)$ & $2(2.4)$ & $0(0.0)$ \\
\hline
\end{tabular}

'Planned reattendances' refers to patients who were recalled for face-to-face assessment by the telephone clinic. 'Unplanned reattendances' refers to patients who reattended without prompting. 


\section{Table 3. Outcomes}

$\begin{array}{llll} & \begin{array}{l}\text { All patients, } \\ \mathbf{n}=\mathbf{1 9 2}\end{array} & \begin{array}{l}\text { Confirmed } \\ \text { or probable } \\ \text { COVID-19, } \\ \mathbf{n = 8 3}\end{array} & \begin{array}{l}\text { Unlikely or } \\ \text { uncertain } \\ \text { COVID-19, } \\ \mathbf{n = 1 0 9}\end{array} \\ \begin{array}{l}\text { Discharged } \\ \text { back to GP, } \\ \mathrm{n}(\%)\end{array} & 131(68.2) & 96(88.1) \\ \begin{array}{l}\text { Referred to } \\ \text { respiratory }\end{array} & 44(22.9) & 41(49.4) & 3(2.8) \\ \begin{array}{l}\text { COVID-19 } \\ \text { follow-up clinic, } \\ \mathrm{n}(\%)\end{array} & & \\ \begin{array}{l}\text { Referred } \\ \text { to other } \\ \text { secondary care } \\ \text { clinic, } \mathrm{n}(\%)\end{array} & & & \\ \end{array}$

(range 1-6), demonstrating that many low-risk patients could be managed with one follow-up assessment alongside communication of their test result. During the course of follow-up, 23 patients $(12 \%)$ were recalled for face-to-face assessments (referred to as planned reattendances in Table 2). The majority of these patients (18/23) were within the confirmed/probable COVID-19 group, and five recalls led to hospital admissions.

We retrospectively reviewed our EHRS to determine whether patients had 'unplanned reattendances' to ED in relation to their suspected COVID-19 illness (ie attendances that were not prompted by the telephone follow-up clinic), either during or after follow-up (Table 2). We identified nine unplanned ED reattendances $(4.7 \%)$, of which two led to admissions. Of note, six reattending patients had been uncontactable during attempted telephone follow-up. This represented a decrease in unplanned
ED reattendance in comparison with our historical comparator cohort (22.6\%). ${ }^{3}$ When asked during assessments, a small number of patients (5 of 192) reported contact with their GP or NHS 111 during follow-up.

There were no deaths within the cohort. Ongoing care destinations are shown in Table 3. Of patients in the confirmed/ probable COVID-19 group, the majority required ongoing specialist care: $49.4 \%$ were referred to the respiratory COVID-19 clinic and $8.4 \%$ were referred to other secondary care clinics. Reasons for referral to the respiratory clinic were persistent breathlessness or other respiratory symptom in 26 patients $(63.4 \%)$ and abnormality in chest imaging on initial presentation in 15 patients (36.6\%).

The majority of patients in the group with unlikely COVID-19/ uncertain diagnosis were referred back to their GP (88.1\%), with the remainder of these patients requiring referral to other secondary care clinics for management of alternative diagnoses. Three patients from this group, for whom a diagnosis of COVID-19 was felt unlikely but possible and who had persistent breathlessness, were referred to the respiratory COVID-19 clinic for further investigation. Formal patient feedback using an SMS survey (sent $\geq 3$ weeks after initial attendance) had a low uptake of $<5 \%$. Informal feedback from patients and other stakeholders such as ED clinicians and GPs was positive.

Recognising that the prevalence of acute COVID-19 (diagnosed at the relevant ED attendance) was low in our pilot cohort, we sought to remeasure our reattendance metrics in a validation cohort from a time of higher COVID-19 incidence. Rates of acute COVID-19 diagnosis in patients managed through the service remained low throughout June-August 2020, but rose in September and October 2020 (supplementary material S1; Fig S2) when rates of positive swabs were $22.5 \%$ and $53.7 \%$ respectively. We carried out a focused evaluation of patients managed through the service from 1 September - 23 October 2020 ( $n=134$, Table 4), of whom $35 \%(n=47)$ had a positive swab for SARSCoV-2. Median age, gender distribution and oxygen saturations were similar to those of the initial cohort, while median C-reactive

Table 4. Validation cohort characteristics and trajectories; emergency department attendances 1 September 23 October 2020

\section{Demographics}

Age, median (IQR)

Female, $\mathrm{n}(\%)$

\section{Characteristics at ED presentation}

Oxygen saturation, \%, median (IQR)

C-reactive protein, $\mathrm{mg} / \mathrm{L}$, median (IQR)

\section{Cohort trajectories}

Number of telephone encounters, median (range)

Total reattendances, $\mathrm{n}(\%)$

Planned reattendances, $\mathrm{n}(\%)$

Planned reattendances leading to admissions, $\mathrm{n}(\%)$

Unplanned reattendances, $\mathrm{n}$ (\%)

Unplanned reattendances leading to admissions, $\mathrm{n}(\%)$

$\begin{array}{ll}\text { All patients, } & \begin{array}{l}\text { COVID-19 positive, } \\ \mathbf{n}=134\end{array} \\ \mathbf{n}=\mathbf{4 7}\end{array}$

COVID-19 negative or swab not done, $\mathrm{n}=87$

$37(27-53)$

$45(51.7)$

$\begin{array}{lll}97.5(96-99) & 96(95.5-98) & 96(96-98) \\ 8.4(1.3-25.4) & 11(3.7-24.7) & 6.9(0.6-25.6)\end{array}$

$1(1-8) \quad 2(1-8) \quad 1(1-5)$

$12(9.0)$

$7(14.6)$

$5(5.7)$

$5(3.7)$

$3(6.2)$

$2(2.3)$

$2(1.5)$

$2(1.3)$

$-$

$7(5.2)^{a}$

$4(8.3)^{b}$

$3(3.4)$

3 (2.2)

$3(6.2)$

Missing data: oxygen saturation (2) and C-reactive protein (12). ${ }^{a}$ of which five had been uncontactable by the telephone clinic; ${ }^{b}$ of which two had been uncontactable; $\mathrm{ED}=$ emergency department; IQR = interquartile range. 
protein level was higher $(8.4 \mathrm{mg} / \mathrm{L}$ vs $2.2 \mathrm{mg} / \mathrm{L} ; \mathrm{p}=0.0005$, MannWhitney $U$ test). Again, we determined reattendances to ED, and stratified these into planned (prompted by the follow-up service) and unplanned. There were 12 reattendances, of which 5 (3.7\%) were planned and 7 (5.2\%) were unplanned (Table 4). Focusing on patients who had tested positive for COVID-19, there were 3 (6.2\%) planned reattendances of which 2 led to admissions, and $4(8.3 \%)$ unplanned reattendances of which 3 led to admissions. The unplanned reattendance rates of 5.2\% (total cohort) and $8.3 \%$ (COVID-19-positive cohort) were comparable to the $4.7 \%$ measured in our pilot cohort and remained lower than the $22.6 \%$ in the historical comparator cohort. ${ }^{3}$

\section{Future directions}

We note that similar remote follow-up pathways or 'virtual wards' have been successfully developed at a number of UK centres for management of COVID-19, incorporating innovations such as use of point-of-care ultrasound in the community to inform remote assessments. ${ }^{11}$ North American centres have extended remote monitoring of COVID-19 patients to automated text message monitoring systems, able to activate clinician telemedicine assessments when necessary. ${ }^{12}$

We suggest that these pathways may contribute to hospital resilience during further potential pandemic waves. When we retrospectively compared our cohort with the cohort who attended the ED at our centre during April 2020 (prior to initiation of the follow-up clinic), ${ }^{3}$ we identified a decrease in unplanned ED reattendances: $4.7 \%$ versus $22.6 \%$. We recognised that this historical comparison was limited by the lower prevalence of acute COVID-19 during the tail of the spring wave of the pandemic. However, we validated our lower unplanned reattendance rates, in a second cohort managed by the remote follow-up service during the upturn of the autumn-winter pandemic wave (35\% of whom tested positive for COVID-19), who had an unplanned reattendance rate of $5.2 \%$ (8.3\% in those testing positive for COVID-19).

We also propose that rapid follow-up still provides an important service to patients who test negative for COVID-19: ensuring rapid communication of test results, allowing assessment of whether a diagnosis of 'probable COVID-19' should be made despite a negative test result, or contributing to identification of an alternative diagnosis and ensuring that patients are referred appropriately. In this way, rapid remote follow-up provides care for unselected patients presenting with symptoms suspicious of COVID-19, and ensures quality of ongoing management (and therefore fewer unplanned ED reattendances) regardless of test result.

There are other limitations to our findings; there may be other contributing factors to lower reattendance rates in more recent cohorts, for example increased public knowledge about COVID-19 symptoms or increased accessibility of other community healthcare providers. Nonetheless, our evaluation suggests that rapid remote follow-up is a framework by which repeated unplanned ED reattendance could be reduced, by ensuring that patients are adequately supported in the community. This could be applicable to both COVID-19 and other conditions. Studies have shown that telephone follow-up can identify and address unmet health needs for older patients discharged from the ED setting,,$^{13}$ and improves patient satisfaction in unselected cohorts discharged from medical wards. ${ }^{14}$

Our evaluation has identified aspects of our practice to build on. Our qualitative experience was that pulse oximetry enhanced telephone assessment of patients, particularly when coupled with simple exercise tests such as 1 minute of sit-to-stand. ${ }^{15} \mathrm{~A}$ cumulative total of $15.1 \%$ of patients in our cohort were using home pulse oximetry, but clinician uptake of offering pulse oximetry to patients on discharge from the ED was low ( $4.2 \%$ of patients used provided oximeters, $10.9 \%$ used their own device). We required ED clinicians to apply specific criteria identifying patients as eligible for provision of a pulse oximeter. We note that other ambulatory COVID-19 services have provided all patients with pulse oximetry, ${ }^{11}$ and minimising clinical gatekeeping could enhance uptake of this inexpensive but clinically valuable intervention.

As the service became established, we began to take direct referrals from GPs managing patients with suspected COVID-19 in the community, and enhancing this alternative referral pathway may be a valuable mechanism by which primary and secondary care can provide joined-up care for ambulatory patients. Many patients required onward referral to secondary care clinics due to persistent symptoms. This may indicate that patients attending the ED were more likely to lie at the moderate-severe end of the COVID-19 spectrum, even if they did not require admission, alongside the fact that some initial ED attendances were due to 'long-tail' persistent COVID-19 symptoms.

We propose that the framework we have developed for rapid remote follow-up represents a robust mechanism by which acute hospitals can safely manage patients with suspected COVID-19 in the community following ED discharge. We suggest that sharing the learning from development of such services across UK centres should be a key consideration in preparedness for further pandemic waves.

\section{Supplementary material}

Additional supplementary material may be found in the online version of this article at www.rcpjournals.org/clinmedicine: S1 - Supplementary tables and figures.

\section{Acknowledgements}

The authors would like to acknowledge the clinicians working in the UCLH departments of emergency medicine, infectious diseases, respiratory medicine and acute medicine, who contributed to the clinical care of this cohort.

\section{References}

1 Emanuel EJ, Persad G, Upshur R et al. Fair allocation of scarce medical resources in the time of Covid-19. N Engl J Med 2020;382:2049-55.

2 Berlin DA, Gulick RM, Martinez FJ. Severe COVID-19. N Engl J Med 2020 [Epub ahead of print].

3 Lanham D, Roe J, Chauhan A et al. COVID-19 emergency department discharges - an outcome study. Clin Med 2021;21: in press.

4 Rayner C, Lokugamage A, Molokhia M. Covid-19: Prolonged and relapsing course of illness has implications for returning workers. BMJ Opinion 2020. https://blogs.bmj.com/bmj/2020/06/23/covid19-prolonged-and-relapsing-course-of-illness-has-implications-forreturning-workers

5 Carfi A, Bernabei R, Landi F. Persistent symptoms in patients after acute COVID-19. JAMA 2020;324:603-5.

6 Chen J, Wu J, Hao $S$ et al. Long term outcomes in survivors of epidemic influenza A (H7N9) virus infection. Sci Rep 2017;7:17275.

7 Ngai JC, Ko FW, Ng SS et al. The long-term impact of severe acute respiratory syndrome on pulmonary function, exercise capacity and health status. Respirology 2010;15:543-50. 
8 Batawi S, Tarazan N, Al-Raddadi R et al. Quality of life reported by survivors after hospitalization for Middle East respiratory syndrome (MERS). Health Qual Life Outcomes 2019;17:101.

9 NHS Health Research Authority. Is my study research? NHS, 2020. www.hra-decisiontools.org.uk/research [Accessed 27 November 2020].

10 Watson J, Whiting PF, Brush JE. Interpreting a covid-19 test result. BMJ 2020;369:m1808.

11 Thornton J. The "virtual wards" supporting patients with covid-19 in the community. BMJ 2020;369:m2119.

12 Morgan AU, Balachandran M, Do D et al. Remote monitoring of patients with Covid-19: design, implementation, and outcomes of the first 3,000 patients in COVID Watch. NEJM Catal Innov Care Deliv 2020:10.1056/CAT.20.0342.

13 Poncia HD, Ryan J, Carver M. Next day telephone follow up of the elderly: a needs assessment and critical incident monitoring tool for the accident and emergency department. J Accid Emerg Med 2000;17:337-40.

14 Braun E, Baidusi A, Alroy G, Azzam ZS. Telephone follow-up improves patients satisfaction following hospital discharge. Eur ] Intern Med 2009;20:221-5.

15 Morita AA, Bisca GW, Machado FVC, Hernandes NA, Pitta F, Probst VS. Best protocol for the sit-to-stand test in subjects with COPD. Respir Care 2018;63:1040-9.

Address for correspondence: Dr Sarah Logan, Hospital for Tropical Diseases, 2B Maple House, 149 Tottenham Court Road, London W1T 7DN, UK.

Email: sarah.logan4@nhs.net

Twitter:@sarahlogan76 